Paidéia, 2003, 12(24), 185-193

\title{
DESENVOLVENDO HABILIDADES DE SOLUÇÃO DE PROBLEMAS INTERPESSOAIS NO ENSINO FUNDAMENTAL ${ }^{1}$
}

\author{
Dâmaris Simon Camelo Borges ${ }^{2}$ \\ FFCLRP - Universidade de São Paulo \\ Edna Maria Marturano \\ FMRP - Universidade de São Paulo
}

\begin{abstract}
RESUMO: Tem sido demonstrada uma relação inversa entre habilidades de solução de problemas interpessoais e comportamentos inibidos ou impulsivos em crianças. Considerando as freqüentes referências a problemas de comportamento em sala de aula, este trabalho teve por objetivo verificar a eficácia do currículo "Eu Posso Resolver Problemas", para desenvolvimento destas habilidades. Participaram do estudo 55 alunos de 1" série, divididos em dois grupos: Grupo de Intervenção $(n=31)$ e Grupo de Comparação $(n=24)$. As crianças que passaram pelo programa melhoraram seu desempenho na geração de soluções para problemas interpessoais. Os alunos que inicialmente mais se envolviam em conflitos diminuíram sua participação nessas ocorrências ao longo do programa.
\end{abstract}

Palavras-chave: habilidade de solução de problemas interpessoais; criança; prevenção.

\section{DEVELOPING INTERPERSONAL PROBLEM SOLVING IN THE ELEMENTARY SCHOOL}

ABSTRACT: An inverse relationship between interpersonal problem solving abilities and inhibited or impulsive behaviors has been demonstrated. Taking into account the frequently reported disciplinary problems in classrooms, this study examined the efficacy of the "I Can Problem Solve" curriculum for the development of these abilities. Fifty-five first-grade students in an elementary public school participated. Two groups were formed: intervention group $(n=31)$ and comparison group $(n=24)$. The children who participated in the program improved their ability for generation of alternative solutions for interpersonal problems as compared to their colleagues who did not participate in the program. Students who initially engaged the most in interpersonal conflicts reduced their participation in these events.

Key-words: interpersonal problem solving; child; prevention.

Com a crescente preocupação com os problemas sociais e emocionais de crianças e adolescentes e a necessidade de preparar estes estudantes para uma participação ativa na sociedade, o papel do professor se alargou; se antes ele tinha uma responsabilidade pedagógica voltada predominantemente para o domínio dos conhecimentos sobre a língua, as ciências e o mundo físico e social, agora tem que se preocupar com o crescimento social, moral e emocional dos seus alunos, sendo que muitas vezes não se sente e não está preparado para isto (Adalbjarnardottir \& Selman, 1997).

${ }^{1}$ Artigo recebido para publicação em 08/2002; aceito em 10/2002

${ }^{2}$ Endereço para correspondência: Damaris Simon Camelo Borges, Rua Francisco Riccioni, 783, Ribeirânia, Ribeirão Preto, SP, Cep 14096-400, e-mail damaris@botelhoborges.com.br
Em um levantamento realizado por Del Prette e Del Prette (1998), em duas cidades do estado de São Paulo, concluiu-se dos relatos dos professores, entre outros aspectos: a) a ocorrência de vários conflitos em sala de aula, bem como de excessos e déficits interpessoais; b) a dificuldade dos professores em lidar com esses conflitos; c) o uso de estratégias frente a essas ocorrências que refletiam um entendimento equivocado do manejo de contingências e o apelo excessivo a contingências aversivas e outras ineficazes. Os dados desse estudo indicam dificuldades interpessoais dos alunos e dos professores, e estas, inclusive, contribuem para exacerbar os conflitos existentes.

A WHO, em documento elaborado pela Divi- 
são de Saúde Mental (1994), apregoa o ensino das habilidades sociais como prevenção primária do uso de drogas, da gravidez na adolescência e da violência, bem como para a prevenção da AIDS, promoção da auto-estima e da auto-confiança. Considerando a relevância das habilidades sociais, o documento diz que o ensino delas deverià ser colocado à disposição de todas as crianças e adolescentes nas escolas, e se possível em idades precoces, antes que padrões de comportamento negativos comecem a se estabelecer.

Nesta investigação, focaliza-se o escolar iniciante e seu comportamento em sala de aula. Partindo da definição da WHO (1994), de habilidades sociais como um conjunto de comportamentos positivos e adaptativos, entre o conjunto delas, optou-se por trabalhar as habilidades de solução cognitiva de problemas interpessoais (HSPI), situadas no campo da cognição social (Romero, 1995).

Segundo Elias e Tobias (1996), um dos principais fatores a colocar uma criança em risco é a impulsividade, que maximiza o perigo e prejudica a ela mesma e aos outros, também deteriorando as relações com pares e adultos. Quando a criança aprende a tentar resolver os problemas e repensar, ela começa a acreditar que pode tomar decisões e resolver suas dificuldades.

Shure (1992) verificou que a partir dos quatro anos é possível detectar diferenças nas HSPI, e que os escores em habilidades de solução cognitiva de problemas interpessoais parecem ser importante preditor do nível de ajustamento e de competência interpessoal dos indivíduos. Essas habilidades são compostas de dois processos básicos: oferecer soluções alternativas para problemas com pares ou autoridade e antecipar o potencial das consequiências na ação interpessoal.

No estudo das associações entre habilidades interpessoais e ajustamento em crianças tem sido demonstrado que: a) habilidades de solução de problemas interpessoais estão associadas a melhor enfrentamento do stress, redução de comportamentos impulsivos ou inibidos (Shure, 1992) e maior competência nas relações interpessoais na adolescência (Elias \& Tobias, 1996); b) habilidades de solução de problemas interpessoais podem ser ensinadas (Elias \& Clabby, 1992; Shure, 1992).

Um Programa para Ensino de Habilidades de Solução de Problemas Interpessoais na Sala de Aula
O ensino das habilidades de solução de problemas interpessoais nas escolas encontra apoio em Elias e Tobias (1996) e no documento da WHO, pela Divisão de Saúde Mental (1994), que situam no meio escolar o público alvo ideal, ao considerar que um dos principais papéis da escola é a socialização.

Refletindo sobre a importância do ensino das habilidades de solução de problemas interpessoais, principalmente na prevenção primária de dificuldades adaptativas, pensou-se em aplicar e avaliar um programa de intervenção na escola pública. Optouse pelo programa proposto por Shure (1992) para ser aplicado em sala de aula, chamado de ICPS Interpersonal Child Problem Solving ou I Can Problem Solve - em português, EPRP - Eu posso resolver problemas.

Mediante consentimento da autora, o programa ICPS / Kindergarten \& Primary Grades (Shure, 1992) foi traduzido, com adaptações de conteúdo à nossa cultura. Para verificar os efeitos da intervenção, havia a necessidade de o programa ser avaliado, pois se efetivamente as habilidades podem ser aprendidas, ele se constitui em um recurso para melhorar as relações das crianças nas situações interpessoais problemáticas.

\section{Objetivos}

A investigação teve por objetivo geral verificar a eficácia do programa de intervenção I Can Problem Solve (Shure, 1992), de ensino de habilidades de solução de problemas interpessoais, em situação de sala de aula no ensino fundamental. Seus objetivos específicos foram: (a) comparar as habilidades de solução de problemas interpessoais (HSPI) nas crianças que cumpriram o programa, antes e depois da intervenção; (b) comparar as HSPI entre as que passaram e que não passaram pelo programa. Como um objetivo adicional, procurou-se caracterizar a sala de aula quanto à ocorrência de conflitos interpessoais ao longo da aplicação do programa.

\section{Método}

\section{Contexto da pesquisa}

Essa pesquisa foi realizada na Escola Municipal de Ensino Fundamental "Professor Jarbas Massullo", situada no bairro Parque São Sebastião, 
localizado na periferia da cidade de Ribeirão Preto. O levantamento sócio-cultural da escola indicou que, em sua maioria, as crianças vivem em famílias nucleares, com renda familiar até cinco salários mínimos, sendo que poucos pais ultrapassaram o nível do ensino fundamental.

\section{Participantes}

Participaram da pesquisa 55 alunos de $1^{*}$ série distribuídos em dois grupos. O GI - Grupo de Intervenção, foi constituído pela totalidade dos alunos de uma classe, em número de 31 , sendo 16 meninas e 15 meninos, com idade variando, no início da intervenção, entre 6 anos e 3 meses e 7 anos e 9 meses (média $=6$ a $9 \mathrm{~m})$. O GC - Grupo de Comparação, foi composto por 24 alunos de três classes de $1^{\prime}$ série da mesma escola, selecionados por idade, sendo 14 meninas e 10 meninos, com idade inicial variando entre 6 anos e 4 meses e 7 anos e 9 meses (média $=7 \mathrm{a} 1 \mathrm{~m}$ ).

\section{Materiais $e$ instrumentos}

Para fim de coleta de dados foram utilizados dois recursos:

a) Sondagem das habilidades de solução de problemas interpessoais - Foi utilizado o PIPS Preschool Interpersonal Problem Solving (Shure, 1990). Esse procedimento, indicado para crianças de 4 a 8 anos, consiste em a criança dar solução a diferentes situações problema com companheiros e mães separadamente. $\mathrm{O}$ procedimento foi planejado para evocar o maior número possível de soluções diferentes por parte da criança, conforme ela se depara com variações da mesma situação. Os problemas interpessoais apresentados se baseiam em duas situações de conflito: entre crianças, trata-se da posse de um brinquedo; entre uma criança e sua mãe, o foco é o dano a uma propriedade desta última.

b) Diário de campo - Foi organizado um diário de campo, com a finalidade principal de documentar problemas interpessoais que ocorriam no dia a dia da sala de aula do Grupo de Intervenção. Nesse diário foram registradas ocorrências como: (a) conflitos e outras situações problemáticas entre os alunos, observadas pela profesșora ou relatadas por eles; (b) situações de conflito entre a professora e um aluno ou grupo de alunos. Esses fatos foram registrados pela professora através de anotações breves, em sala de aula, no momento em que ocorriam. Imediatamente após o término da aula, as anotações eram complementadas com os pormenores de cada acontecimento registrado e digitadas em arquivo de texto. O diário de campo foi escrito de fevereiro a outubro.

\section{Procedimento de coleta de dados}

Todas as fases da investigação foram conduzidas pela primeira autora, que era a professora regente da classe de $1^{*}$ série que passou pela intervenção. Foi solicitado à Secretaria Municipal de Ensino e às mães permissão para que se pudesse proceder ao estudo. Mediante a aceitação das mães, foi preenchido o Termo de Consentimento Informado; procedeu-se à avaliação pré-intervenção; desenvolveu-se o processo de intervenção; por fim as crianças foram reavaliadas nas mesmas habilidades. $O$ procedimento foi aplicado no Grupo de Intervenção em 2000; as avaliações do Grupo de Comparação foram feitas em 2001, nos mesmos meses em que GI foi avaliado.

\section{O Programa de intervenção}

O programa foi ministrado em duas a três sessões por semana, com a duração de 15 a 20 minutos, no período de abril a outubro. A intervenção compreendeu os três componentes incluídos no manual do programa (Shure, 1992), indicados para alunos do ensino fundamental. O primeiro é um currículo com 68 lições formais, organizado em três partes - Habilidades Prévias para Solução de Problemas, Habilidade de Solução de Problemas, Processo de Solução Cognitiva de Problemas Interpessoais. O segundo componente é um conjunto de sugestões para incorporar os princípios do EPRP no cotidiano da sala de aula e no currículo regular. $O$ terceiro é uma proposta de "Diálogo EPRP", matriz de diálogo segundo as etapas de solução de problemas ensinadas no currículo, que ajuda a treinar as habilidades de pensamento recém adquiridas, negociar os relacionamentos e lidar com as frustrações.

\section{Procedimento de análise de dados}

Análise das respostas ao PIPS - No manual (Shure, 1990) são fornecidos critérios para classificação das respostas como "solução" ou "não solução". Um outro sistema de classificação guia o exa- 


\section{Dâmaris Simon Camelo Borges}

minador no julgamento adicional que deve fazer, afirmando se é uma solução nova, diferente de todas as que a criança forneceu até então. Uma resposta relevante é aquela que atende aos dois requisitos. $O$ escore final é a taxa de relevância, que consiste na razão entre o número de soluções relevantes e o total de respostas dadas.

A classificação das respostas foi feita por uma auxiliar de pesquisa, psicóloga, através de um procedimento cego, ou seja, sem ter informação sobre a composição dos grupos ou do momento em que as avaliações aconteceram.

Análise do Diário de Campo - A análise dos registros do Diário de Campo focalizou as ocorrências de conflitos interpessoais e as intervenções da professora nessas situações. Para permitir a delimitação clara das ocorrências de conflito, foram definidas sete classes: Agredindo fisicamente / sendo agredido / agressão recíproca; Desobedecendo à ordem da professora; Provocando; Discutindo; Infringindo uma regra da escola ou da sala de aula; Mexendo no material alheio; Disputando um objeto.

Como forma de aferir a fidelidade da implementação das duas estratégias para a generalização do EPRP - Diálogo EPRP e incorporação dos conceitos ao cotidiano da sala de aula - a intervenção da professora em cada ocorrência de conflito foi qualificada, segundo critérios propostos no manual do programa, como estratégia completa, incompleta, esclarecimento da situação ou ausência de estratégia de generalização do EPRP.

Fidedignidade - Testes de acordo entre a auxiliar de pesquisa e a primeira autora foram feitos sobre a classificação das respostas ao PIPS e dos conflitos registrados no diário de campo. Foram utilizados para cálculos de acordo dados de quatro crianças que saíram da classe por razões diversas, não participando do programa EPRP até o final. As porcentagens de classificação concordante foram: no PIPS, 74\%, 92\%, 68\%, 88\%; na classificação de conflitos, $62 \%, 80 \%, 86 \%$ e $100 \%$

\section{Resultados}

\section{Taxa de respostas relevantes: habilidade de ge- rar soluções para problemas interpessoais}

A taxa de respostas relevantes foi tomada como indicador de melhora na habilidade de gerar soluções alternativas para problemas interpessoais. Uma ANOVA $2 \times 2$ (grupo x sexo) antes da intervenção mostrou não existir diferença entre os grupos, mas haver entre sexos $(F=4,156, p=0,047)$, tendo as meninas inicialmente taxas mais altas de respostas relevantes.

Para verificar o efeito da intervenção, foram utilizados procedimentos estatísticos visando à verificação de efeitos de tratamentos, baseados em delineamento de comparação entre grupos com medidas pré e pós-intervenção (Webster-Stratton \& Hammond, 1997). Empregou-se uma ANCOVA tendo como variável dependente a taxa de respostas relevantes na segunda avaliação, como fatores as condições grupo e sexo e como co-variante a taxa de respostas relevantes na primeira avaliação. Essa análise mostrou efeito significativo associado ao fator grupo $(F=10,927, p=0,002)$, tendo o Grupo de Intervenção taxa média mais alta de respostas relevantes. Esse resultado indica que a intervenção teve o efeito esperado de elevar a capacidade de geração de soluções alternativas para problemas interpessoais.

$\mathrm{Na}$ segunda avaliação, não foram detectadas diferenças entre sexos.

Testes $t$ para grupos emparelhados foram aplicados aos dados de cada grupo para verificar diferenças nas taxas de resposta relevante entre a primeira e a segunda avaliação. Foi detectada melhora significativa no Grupo de Intervenção $(t=$ $4,266, p<0.0001$ ), mas não no Grupo de Comparação $(t=1,466, p=0,16)$, indicando que foi a melhora no Grupo de Intervenção que ocasionou as diferenças encontradas após a apliteção do programa.

No manual do PIPS (Shure, 1990) a taxa de relevância igual ou inferior a 0.60 é considerada como indicativa de dificuldades de comportamento; de acordo com esse critério, o Grupo de Intervenção tinha 8 crianças com dificuldades antes da intervenção e esse número caiu para 3 após a intervenção; no Grupo de Comparação, o número variou de 4 para 6 da $1^{\mathrm{a}}$ para a $2^{\mathrm{a}}$ avaliação.

\section{As classes de resposta: soluções preferidas}

A fim de verificar variações nas classes de solução apresentadas pelas crianças ao PIPS, os dados das crianças de cada grupo foram agrupados, 
considerando-se todas as respostas de solução, inclusive as repetidas. Apenas os resultados relativos aos problemas com companheiros são apresentados, na Tabela 1.

Tabela 1: Classes de solução apresentadas pelas crianças para conflitos entre pares, nas duas avaliações. Dados agrupados de todos os participantes de cada grupo.

\begin{tabular}{|c|c|c|c|c|}
\hline \multirow[t]{2}{*}{ Classes de solução } & \multicolumn{2}{|c|}{$\begin{array}{c}\text { Grupo de Intervenção } \\
(\mathbf{n = 3 1})\end{array}$} & \multicolumn{2}{|c|}{$\begin{array}{c}\text { Grupo de Comparação } \\
(n=24)\end{array}$} \\
\hline & $\begin{array}{c}\text { Pré- } \\
\text { intervenção }\end{array}$ & $\begin{array}{c}\text { Pós- } \\
\text { intervenção }\end{array}$ & $\begin{array}{c}\text { Início da } \\
1^{\mathrm{a}} \text { série }\end{array}$ & $\begin{array}{l}\text { Fim da } \\
1^{\text {a série }}\end{array}$ \\
\hline Pedir permissão & 58 & 25 & 27 & 24 \\
\hline Pedir por favor & 13 & 21 & 10 & 12 \\
\hline Pedir permissão para pegar emprestado & 71 & 88 & 51 & 57 \\
\hline Dizer: "vou tomar cuidado" & 0 & 0 & 1 & 1 \\
\hline Brincar juntos & 41 & 41 & 33 & 37 \\
\hline Brincar revezando & 18 & 28 & 7 & 7 \\
\hline Justiça & 0 & 13 & 0 & 4 \\
\hline Fazer o outro sentir-se culpado & 0 & 8 & 0 & 1 \\
\hline Negociação direta & 10 & 19 & 8 & 15 \\
\hline Convite & 1 & 5 & 2 & 6 \\
\hline Negociação envolvendo um objeto & 13 & 31 & 13 & 13 \\
\hline Negociação envolvendo prestação de serviço & 2 & 5 & 2 & 2 \\
\hline Intervenção de autoridade & 20 & 21 & 22 & 17 \\
\hline Trapaça & 1 & 3 & 10 & 5 \\
\hline Despistar & 10 & 10 & 8 & 20 \\
\hline Manipulação psicológica & 1 & 1 & 4 & 1 \\
\hline Manipulação da amizade & 11 & 5 & 5 & 8 \\
\hline Comentário sobre o brinquedo & 2 & 1 & 1 & 1 \\
\hline Fazer $o$ outro sentir pena & 7 & 16 & 1 & 7 \\
\hline Ficar brava & 0 & 0 & 1 & 1 \\
\hline Esperar & 5 & 23 & 4 & 7 \\
\hline Pegar à força & 27 & 9 & 16 & 14 \\
\hline Surrupiar & 20 & 7 & 8 & 5 \\
\hline Agressão física & 10 & 6 & 10 & 6 \\
\hline Agressão por parte de terceiros & 0 & 1 & 1 & 0 \\
\hline Resposta específica p/brinquedo & 1 & 0 & 1 & 0 \\
\hline Dano à propriedade - ameaças & 0 & 4 & 1 & 0 \\
\hline Comandos & 2 & 1 & 6 & 4 \\
\hline Planos para o future & 6 & 0 & 2 & 0 \\
\hline
\end{tabular}

Verifica-se no Grupo de Intervenção que, a par com um aumento generalizado em diversas classes de solução, respostas anti-sociais como pegar à força, surrupiar e agressão física diminuíram, assim como soluções de manipulação da amizade. Houve aumento nas soluções pertencentes às classes de justiça, revezamento e negociação.

No Grupo de Comparação foram observadas tendências semelhantes, quanto ao aumento de tentativas de negociação ativa e diminuição de soluções agressivas. Porém as diferenças aqui são no geral menos pronunciadas que no grupo que passou pelo programa. No grupo de comparação, o aumento das classes "despistar" e "manipulação da amizade" pode ser indício da menor sensibilização para os sentimentos dos outros.

\section{Conflitos registrados no Diário de Campo}

Os relatos de conflitos foram agrupados em seis blocos de 24 dias letivos. Esse agrupamento foi feito de modo que o periodo de férias escolares incidisse entre blocos e não dentro de um deles. Os resultados individuais se encontram na Tabela 2 . 
Tabela 2: Número de participações de cada criança nos conflitos em sala de aula, registrados no diário de campo.

\begin{tabular}{lcccccc}
\hline Criança & 2, b & \multicolumn{6}{l}{ Freqüência } & (períodos de 24 dias letivos) \\
\cline { 2 - 7 } & $\mathbf{1}$ & $\mathbf{2}$ & $\mathbf{3}$ & $\mathbf{4}$ & $\mathbf{5}$ & $\mathbf{6}$ \\
\hline Wesley & 27 & 6 & 8 & 4 & 18 & 13 \\
Danila & 23 & 13 & 8 & 11 & 22 & 12 \\
Ednilson & 16 & 8 & 12 & 13 & 26 & 9 \\
Valéria & 15 & 3 & 2 & 4 & 3 & 3 \\
Iago & 12 & 1 & 5 & 5 & 9 & 8 \\
Eliana & 9 & 8 & 3 & 2 & 9 & 7 \\
Thacia & 9 & 1 & 5 & 0 & 2 & 2 \\
Paulo & 7 & 3 & 2 & 2 & 2 & 2 \\
Vanderilson & 7 & 5 & 1 & 2 & 4 & 4 \\
Lyan & 6 & 14 & 10 & 1 & 6 & 8 \\
Fábia & 5 & 0 & 1 & 4 & 8 & 2 \\
Fausto & 3 & 14 & 6 & 4 & 25 & 4 \\
Vander & 3 & 1 & 4 & 3 & 10 & 3 \\
Márcio & 3 & 2 & 6 & 15 & 6 & 5 \\
George & 3 & 7 & 4 & 7 & 6 & 9 \\
Nanda & 1 & 0 & 0 & 0 & 2 & 0 \\
Vanessa & 1 & 0 & 2 & 0 & 4 & 1 \\
Marlon & 1 & 8 & 2 & 7 & 5 & 10 \\
Raphaella & 1 & 1 & 2 & 0 & 2 & 0 \\
Lina & 0 & 0 & 0 & 1 & 0 & 0 \\
Fabiana & 0 & 1 & 1 & 2 & 4 & 0 \\
Júlia & 0 & 0 & 4 & 4 & 3 & 6 \\
Laurinda & 0 & 4 & 2 & 2 & 0 & 0 \\
Leonildo & 0 & 2 & 1 & 7 & 0 & 5 \\
Mário Humberto & 0 & 0 & 0 & 0 & 3 & 0 \\
Juliana & 0 & 4 & 8 & 0 & 3 & 2 \\
Letícia & 0 & 2 & 1 & 1 & 0 & 2 \\
Lucilene & 0 & 1 & 5 & 1 & 1 & 0 \\
Helena & 0 & 5 & 9 & 5 & 4 & 3 \\
Hélio & 0 & 0 & 5 & 2 & 4 & 7 \\
Danilo & 0 & 0 & 20 & 6 & 23 & 18 \\
\hline TOTAL & $\mathbf{1 5 2}$ & $\mathbf{1 1 4}$ & $\mathbf{1 3 9}$ & $\mathbf{1 1 5}$ & $\mathbf{2 1 4}$ & $\mathbf{1 4 5}$ \\
\hline & & & & & & \\
\hline
\end{tabular}

${ }^{a}$ Nomes fictícios.

${ }^{b} \mathrm{Em}$ ordem decrescente, pelo número de conflitos registrados no primeiro período.

'Período pós-férias de julho.

Observa-se na Tabela 2 que o total de conflitos não diminui entre o período 1 (anterior à intervenção) e o período 6 (posterior à intervenção), mas chama a atenção a diminuição de ocorrências do período 1 ao $4 \mathrm{e} o$ aumento que ocorreu no período 5 (que sucedeu as férias). Os resultados individuais indicam que as crianças com maior participação em conflitos no $1^{\circ}$ período obtiveram diminuição progressiva até o $4^{\circ} \mathrm{e}$ chegaram ao último com índices mais baixos que os iniciais. Por outro lado, há crianças, como Danilo, que aumentam sua participação em conflitos.

\section{Análise das intervençōes da professora}

A aplicação dos critérios de implementação dos conceitos do EPRP pela professora forneceu os resultados apresentados na Tabela 3. Proxima Página.

Observando a Tabela 3, verifica-se que em apenas $16 \%$ dos conflitos interpessoais em sala de aula houve uma intervenção prática completamente adequada de acordo com os princípios do EPRP. Também pode ser observado que os índices de intervenções adequadas (estratégias completas) e parcialmente adequadas (estratégias incompletas) crescem ao longo do tempo. Inversamente, as intervenções consideradas como apenas uma tentativa de esclarecer a situação, ou onde não houve nenhum tipo de estratégia do EPRP, diminuíram ao longo do período.

\section{Discussão}

O principal objetivo deste estudo foi verificar a eficácia do programa de intervenção EPRP (Shure, 1992) para uma situação de sala de aula na escola elementar. Tendo comparado as habilidades cognitivas de solução de problemas interpessoais nas crianças antes e depois do programa de intervenção e obtido um aumento na taxa de respostas relevantes estatisticamente significante, pode-se dizer que o programa de intervenção é eficaz, para as crianças desse agrupamento sócio-cultural, no sentido de melhorar tais habilidades. A comparação com um grupo que não passou pelo processo de intervenção mostrou indícios de um processo de aprendizagem social em curso no Grupo de Comparação, mas a simples freqüência à primeira série não traz melhoras em niveis estatisticamente significantes.

Também foram observados indícios do efeito positivo do EPRP em outros indicadores, como a diminuição dos casos de crianças com taxa de relevância inferior a 0,60 no PIPS e a redução no número de conflitos envolvendo as crianças 
Tabela 3: Caracterização das interações quanto à qualidade da intervenção.

\begin{tabular}{|c|c|c|c|c|c|c|c|c|}
\hline \multirow{3}{*}{ Período letivo } & \multicolumn{8}{|c|}{ Qualidade da Intervenção } \\
\hline & \multicolumn{2}{|c|}{ Completa } & \multicolumn{2}{|c|}{ Incompleta } & \multicolumn{2}{|c|}{$\begin{array}{c}\text { Esclarece a } \\
\text { situação }\end{array}$} & \multicolumn{2}{|c|}{ Sem EPRP } \\
\hline & $\mathbf{F}$ & $\%$ & $\mathbf{F}$ & $\%$ & $\mathbf{F}$ & $\%$ & $\mathbf{F}$ & $\%$ \\
\hline $15 / 02$ a $20 / 03$ & 0 & 0 & 0 & 0 & 41 & 34 & 80 & 66 \\
\hline $21 / 03$ a $19 / 04$ & 1 & 1 & 1 & 1 & 25 & 30 & 57 & 67 \\
\hline $20 / 04$ a $25 / 05$ & 4 & 4 & 9 & 9 & 22 & 22 & 67 & 66 \\
\hline $26 / 05$ a $29 / 06$ & 19 & 23 & 8 & 10 & 10 & 12 & 45 & 55 \\
\hline $10 / 08$ a $14 / 09$ & 38 & 27 & 30 & 21 & 16 & 11 & 57 & 40 \\
\hline $15 / 09$ a $20 / 10$ & 41 & 35 & 10 & 9 & 8 & 7 & 57 & 49 \\
\hline Total & 103 & 16 & 58 & 9 & 122 & 19 & 363 & 56 \\
\hline
\end{tabular}

consideradas com comportamentos inicialmente mais perturbadores, confirmando o efeito preventivo do EPRP e sua ação benéfica para as mais difíceis. Por outro lado, para algumas crianças o EPRP não parece ter tido efeito favorável.

Considerações podem ser feitas a partir da análise do PIPS e do diário de campo. A análise das classes de respostas dadas pelas crianças ao PIPS no pré e no pós teste, com aumento das classes consideradas pró-sociais e diminuição daquelas consideradas anti-sociais, reforça a afirmação de Shure e Spivack (1982) de que o estilo de pensar e o conteúdo das respostas não estão dissociados e a probabilidade de a pessoa manifestar uma solução mais efetiva vai depender do exercício do processo do EPRP. $O$ aumento das classes que envolvem a negociação, em uma atitude basicamente democrática, e a sensibilização do outro na solução de problemas parecem indícios de que o EPRP estimula a criança a negociar conflitos considerando os diferentes pontos de vista dos envolvidos na situação.

A diminuição de soluções de força, em ambos os grupos, parece refletir uma tendência evolutiva da fase escolar (Chazan, Laing \& Davies, 1994), em que estratégias de agressão manipulativa são substituídas por recursos socialmente mais adequados (Brioso \& Sarria, 1995). Contudo, apesar de nos dois grupos as crianças terem diminuído as soluções consideradas agressivas, no Grupo de Intervenção aumentaram as soluções consideradas como sendo características de negociação e de espera de oportunidades e no Grupo de Comparação aumentaram as soluções características de manipulação.
A pronunciada variação no envolvimento em conflitos, entre o período que antecedeu e o que sucedeu as férias, suscita reflexões. Pode-se pensar em efeitos negativos de uma interrupção do programa, considerando-se que no momento da interrupção as crianças estavam aprendendo a conhecer os sentimentos, mas ainda não tinham aprendido a lidar com eles. Esse efeito pode ter-se somado a um fenômeno típico de pós- férias, em que as professoras encontram seus alunos um tanto mais agitados do que antes do recesso. Nos dois casos, fica reforçada a idéia da necessidade de um suporte constante que propicie múltiplas possibilidades de interação positiva entre iguais e com adultos significativos para a criança, bem como a necessidade de um projeto mais amplo que consiga abarcar os diferentes meios de interação da criança, entre eles a comunidade, a escola e a família (Arbol \& Arangurem, 1995; Brioso \& Sarria, 1995).

Quanto às intervenções da professora, os resultados da análise exploratória que foi feita sugerem um processo de aprendizagem durante a aplicação do programa, apesar de ter havido treino prévio durante o estudo piloto mencionado na introdução. A esse respeito, Adalbjarnardottir e Selman (1997) observaram que programas de intervenção como este exigem uma tomada de consciência dos professores a respeito das próprias habilidades sociais. Sem essa tomada de consciência, além da instrução a respeito dos princípios que norteiam o desenvolvimento infantil, corre-se o risco de associar, de forma simplista, a ação do educador à artificialidade e manipulação do indivíduo (Brioso \& Sarria, 1995). 
Segundo Yeates e Selman (1989) a criança aprende passos de resolução de problemas com relativa facilidade e a alteração das ações e da reflexão cognitiva incita um considerável desconforto para a criança e até mesmo para muitos adultos; portanto, sem que o meio esteja envolvido e dê suporte à implementação do modelo, existem poucas esperanças para a mudança de comportamento.

Como foi enfatizado na introdução, a meta do programa é gerar na criança formas de pensar e usar suas próprias crenças, valores e padrões de resposta na tomada de decisão relacionada aos problemas que surgem. Essa conceituação remete à questão do repertório dos indivíduos, já que a capacidade de agir fica limitada ao repertório de cada um. A flexibilidade de atitudes diante dos problemas que surgem, propiciada pelo desenvolvimento das HSPI, tem seus limites estabelecidos pela diversidade de estratégias de que o indivíduo dispõe, em um determinado momento, para solucionar os problemas.

Em suma, para que obtivéssemos resultados mais efetivos sobre o comportamento seria necessário talvez ampliar o campo de ação do procedimento de intervenção para o âmbito familiar, tanto no sentido de preparar o meio em que a criança vive para dar suporte às mudanças esperadas, quanto no sentido de calibrar expectativas de comportamento do meio escolar com o meio familiar e, talvez, complementar a intervenção com a discussão de valores, considerados universais e que poderiam suprir o repertório das crianças na consideração das alternativas de solução dos problemas interpessoais.

Para finalizar, algo deve ser dito sobre limitações metodológicas do estudo. $O$ registro de conflitos no diário de campo pela própria professora, envolvida duplamente com a proposta de intervenção, restringe o alcance dos dados gerados através desse recurso. A falta de dados de comportamento sobre $o$ Grupo de Comparação é um segundo fator limitante na avaliação dos efeitos do programa sobre o comportamento.

Com essas ressalvas, pode-se dizer que o procedimento de intervenção denominado EPRP melhorou cognitivamente a capacidade de solução de problemas interpessoais em termos quantitativos, quanto à solução alternativa de problemas, e deu indícios de melhora em termos de qualidade de raciocínio, quanto à natureza das soluções escolhidas.
Dentro dos limites metodológicos, os resultados sugerem que as crianças com maior envolvimento em conflitos interpessoais foram as mais beneficiadas pela intervenção. Esse achado animador precisa ser verificado em estudo mais controlado. A persistência e a generalização dos efeitos também são questões que ficam em aberto.

\section{Referências Bibliográficas}

Adalbjarnardottir, S.E \& Selman, R.L (1997). "I feel I received a new vision": An analysis of teacher's professional development as they work with students on interpersonal issues. Teaching and Teacher Education, 13, 409 - 428.

Arbol, L.R.D. \& Arangurem, L.L. (1995). A escola perante a inadaptação social. In: C. Coll; J. Palácios \& A. Marchesi (Orgs.), Desenvolvimento Psicológico e Educação Necessidades Educativas Especiais $e$ Aprendizagem Escolar (pp. 169-179). Porto Alegre: Artes Médicas.

Brioso, A. \& Sarria, E. (1995). Distúrbios de comportamento. In: C. Coll; J. Palácios \& A. Marchesi (Orgs.), Desenvolvimento Psicológico $e$ Educação - Necessidades Educativas Especiais e Aprendizagem Escolar (pp. 157168). Porto Alegre: Artes Médicas.

Chazan, M., Laing, A.F. \& Davies, D. (1994). Emotional and behavioural difficulties in middle childhood. Identification, assesment and intervention in school. London: The Falmer Press.

Del Prette, Z.A.P. \& Del Prette, A. (1998). Desenvolvimento interpessoal e educação escolar: o enfoque das habilidades sociais. São Carlos: Temas em Psicologia.

Elias, M.J. \& Clabby, J.F. (1992). Building social problem - solving skills: guidelines from a school based program. San Francisco: JosseyBass Publishers.

Elias, M. \& Tobias, S.E. (1996). Social problem solving : Interventions in the schaols. New York: The Guilford Press. 
Romero, J.F. (1995). As relações sociais das crianças com dificuldades de aprendizagem. In: C. Coll; J. Palácios \& A. Marchesi (Orgs.), Desenvolvimento psicológico e educação: necessidades educativas especiais e aprendizagem escolar. Porto Alegre: Artes Médicas.

Shure, M.B. (1992). I can problem solve. Illinois: Reserch Press.

Shure, M.B. (1990). The PIPS test: Manual 2nd edition - For 4 to 6 year old children. Philadelphia: Hahnemann University Department of Mental Health Sciences.

Shure, M.B. \& Spivack, G. (1982). Interpersonal problem - solving in young children: A cognitive approach to prevention. American Journal of Community Psychology, 10 (3), 341-356.

Webster-Stratton, C.E \& Hammond, M. (1997). Treating children with early- onset conduct problems: a comparison of child and parent training interventions. Journal of Consulting and Clinical Psychology,. 65, 93-109.

World Health Organization: Division of Mental Health (1994). Life skills education in schools, Geneva.

Yeates, K.O. \& Selman, R.L. (1989). Social competence in the schools: Toward an Integrative developmental model for intervention Developmental Review, 9, 64-100.

\section{APOIO: FAPESP}


194 